\title{
Novel analysis methods of dynamic properties for vehicle pantographs
}

\author{
Andrzej Wilk ${ }^{1, *}$ \\ ${ }^{1}$ Gdansk University of Technology, Faculty of Electrical and Control Engineering, ul. G. Narutowicza 11/12, 80-233, Gdańsk, Poland
}

\begin{abstract}
Transmission of electrical energy from a catenary system to traction units must be safe and reliable especially for high speed trains. Modern pantographs have to meet these requirements. Pantographs are subjected to several forces acting on their structural elements. These forces come from pantograph drive, inertia forces, aerodynamic effects, vibration of traction units etc. Modern approach to static and dynamic analysis should take into account: mass distribution of particular parts, physical properties of used materials, kinematic joints character at mechanical nodes, nonlinear parameters of kinematic joints, defining different parametric waveforms of forces and torques, and numerical dynamic simulation coupled with FEM calculations. In this work methods for the formulation of the governing equations of motion are presented. Some of these methods are more suitable for automated computer implementation. The novel computer methods recommended for static and dynamic analysis of pantographs are presented. Possibilities of dynamic analysis using CAD and CAE computer software are described. Original results are also presented. Conclusions related to dynamic properties of pantographs are included. Chapter 2 presents the methods used for formulation of the equation of pantograph motion. Chapter 3 is devoted to modelling of forces in multibody systems. In chapter 4 the selected computer tools for dynamic analysis are described. Chapter 5 shows the possibility of FEM analysis coupled with dynamic simulation. In chapter 6 the summary of this work is presented.
\end{abstract}

\section{Introduction}

Transfer of the electrical energy from overhead catenary system has to be safe and reliable at different operating conditions. For this purpose a contact force value between the pantograph and the overhead contact line is recommended for different voltage systems of electric railways [1]. A lot of research was realized on design, construction and materials used to make pantographs. In addition such features as: compact profile for saving of space, lower weight, low aerodynamic noise and minimum maintenance are taken into account.

High performance pantograph is usually a single arm pantograph. It consist of a main frame, lower and upper arms, coupling rod, balance rod, pantograph head and drive (Fig.1). The concept of the operation between lower and upper working positions is based on two articulated quadrangles. The first articulated quadrangle includes a part of the main frame, the lower arm, the part of upper arm and the coupling rod. The second one includes a small part of the upper arm, the balance rod, the link element and the upper arm. The moving pantograph is subjected to static and dynamic forces. Hence, the dynamic properties of pantograph are important for its entire working range during both upward and downward movements [2].

The objective of dynamic simulation is to determine the time-related behaviour of all pantograph assembly and analysis of interactions between particular parts (links) in connecting joints $[3,4]$. The dynamic simulation is useful to determine the contact force acting by the collector strips on the contact wire. Computational methods used in dynamic simulation should be coupled with FEM calculations in order to analyse stress acting on the pantograph parts.

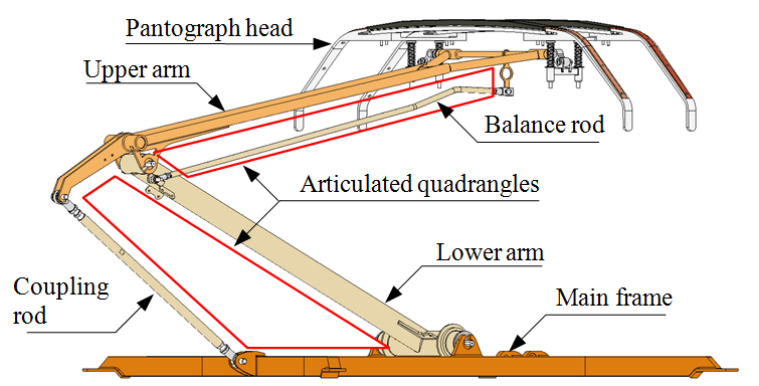

Fig. 1. Railway pantograph as a multibody system with closed loops (two articulated quadrangles).

\section{Methods for formulation of equations}

For mechanical systems only Newton's second law and D'Alembert principle are usually sufficient. However a pantograph consists of numerous rigid-body links (arms, rods etc.) and different joints, hence the formulation of governing equations from Newtonian approach is very

\footnotetext{
* Corresponding author: andrzej.wilk@pg.edu.pl
} 
difficult. For complex mechanical systems Lagrange, Hamilton and Gibbs equations of motion are developed based on energy equations. Computer applications need to derive numerical form of governing equations ready for computer implementation. Automated form of the equations of motion can be derived using Kane's equations [5]. In the past two decades the computational methods used in the dynamic simulation of flexible multibody systems were developed based on the Finite Element Methods (FEM) [6] and Boundary Element Methods (BEM) [7].

\subsection{Lagrange's equation}

Lagrange's equation is derived from energy-state functions. The general form of Lagrange's equation for conservative mechanical system with $k$ degrees of freedom is given by

$$
\frac{\mathrm{d}}{\mathrm{d} t}\left[\frac{\partial L(\dot{\xi}, \xi, t)}{\partial \dot{\xi}}\right]-\frac{\partial L(\dot{\xi}, \xi, t)}{\partial \xi}=f_{\mathrm{k}}, \quad k=1,2, \ldots N
$$

where $L$ is the Lagrangian of the system, $\xi$ is the vector of generalized coordinates (including translational and rotational positions), $f$ represents the generalized forces applied and associated with the constraints.

The diagram of the simplified kinematic system of articulated pantograph with two degrees of freedom is shown in Fig.2. In this kinematic system only angular displacement $\alpha_{1}$ of the lower arm and vertical displacement $y$ of the pantograph head are assumed as degrees of freedom.

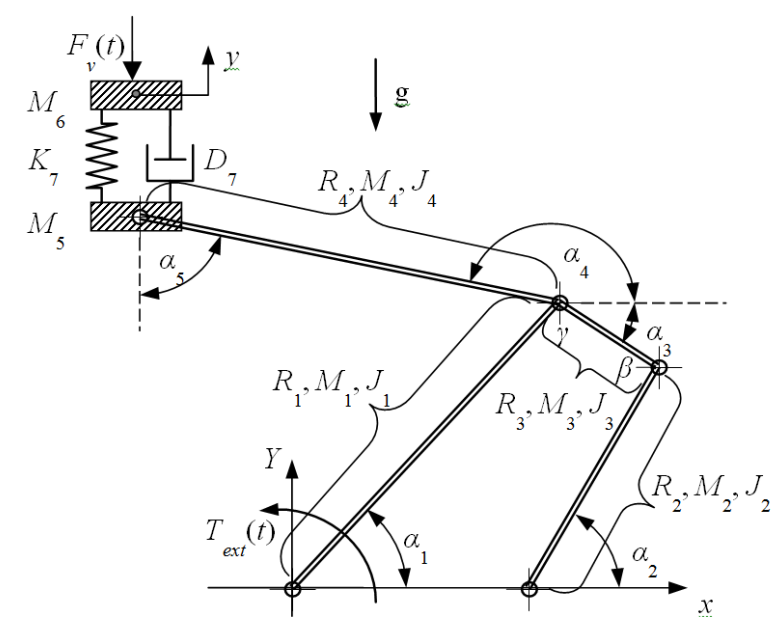

Fig. 2. Kinematic diagram of articulated type pantograph with two degrees of freedom - rotational and translational motion.

For this case the governing equation as system of nonlinear differential equations is [8]

$$
\begin{aligned}
& \frac{\mathrm{d}}{\mathrm{d} t}\left(\frac{\partial L\left(\dot{\alpha}_{1}, \alpha_{1}, \dot{y}, y\right)}{\partial \dot{\alpha}_{1}}\right)-\frac{\partial L\left(\dot{\alpha}_{1}, \alpha_{1}, \dot{y}, y\right)}{\partial \alpha_{1}}+\frac{\partial P_{m}\left(\dot{\alpha}_{1}, \dot{y}\right)}{\partial \dot{\alpha}_{1}}=f_{\alpha}(t), \\
& \frac{\mathrm{d}}{\mathrm{d} t}\left(\frac{\partial L\left(\dot{\alpha}_{1}, \alpha_{1}, \dot{y}, y\right)}{\partial \dot{y}}\right)-\frac{\partial L\left(\dot{\alpha}_{1}, \alpha_{1}, \dot{y}, y\right)}{\partial y}+\frac{\partial P_{\mathrm{m}}\left(\dot{\alpha}_{1}, \dot{y}\right)}{\partial \dot{y}}=f_{y}(t)
\end{aligned}
$$

where $f_{\alpha}$ and $f_{y}$ are the so-called generalized forces resulting from applied torques and forces.
Generalized force $f_{\alpha}\left(t, \alpha_{1}\right)$ can be represented as a superposition of three torques acting on the pantograph

$$
f_{\alpha}\left(t, \alpha_{1}\right)=T_{e x t}\left(t, \alpha_{1}\right)+T_{g}\left(\alpha_{1}\right)+T_{f y}\left(t, \alpha_{1}\right)
$$

where: $T_{\text {ext }}\left(t, \alpha_{1}\right)$ is the torque raising the pantograph, $T_{\mathrm{g}}\left(\alpha_{1}\right)$ is the torque resulting from gravity, $T_{f y}\left(t, \alpha_{1}\right)$ is the torque derived from the contact force $F_{y}(t)$ as a result of catenary interaction. All this torque components are functions of the rotation angle, even when the forces have a fixed value. The explicit matrix form of equation (2) is given by

$$
\left[\begin{array}{cc}
J_{\alpha}\left(\alpha_{1}\right) & k_{y}\left(\alpha_{1}\right) \\
k_{y}\left(\alpha_{1}\right) & M_{6}
\end{array}\right]\left[\begin{array}{c}
\ddot{\alpha}_{1} \\
\ddot{y}
\end{array}\right]=\left[\begin{array}{c}
Q_{\alpha}(t)-h_{\alpha}\left(\alpha_{1}\right) \dot{\alpha}_{1}^{2}-D_{\alpha}\left(\alpha_{1}\right) \dot{\alpha}_{1} \\
Q_{y}(t)-h_{y}\left(\alpha_{1}\right) \dot{\alpha}_{1}^{2}-D_{7} \dot{y}-\frac{y}{K_{7}}
\end{array}\right]
$$

where coefficients $J_{\alpha}\left(\alpha_{1}\right), h_{\alpha}\left(\alpha_{1}\right), k_{y}\left(\alpha_{1}\right), h_{\mathrm{y}}\left(\alpha_{1}\right)$ are very complex functions of the $\alpha_{1}$ rotation angle. They can be determined from physical properties of materials and dimensions of pantograph parts. The substitute coefficient of viscous damping $D_{\alpha}\left(\alpha_{1}\right)$ and dry damping are usually determined using an experimental approach.

\subsection{Hamilton's equation}

The Hamilton principle is related to the Lagrange energy method through transformation called the Legendre transformation. Hamiltonian formalism in the formulation of equations takes into account the total energy of the system. Hamiltonian function $H$ for the system with $n$ degrees of freedom is defined as follows:

$$
\mathrm{H}(\xi, \mathbf{p}, t)=\sum_{\mathrm{i}=1}^{\mathrm{n}} p_{\mathrm{i}} \xi_{\mathrm{i}}-L(\dot{\xi}, \xi, t)
$$

where $p$ is generalized momentum conjugate with $\xi_{\mathrm{i}}$, that is

$$
p_{i}=\frac{\partial \mathrm{L}(\dot{\xi}, \xi, t)}{\partial \xi_{i}} \quad i=1, \ldots, n
$$

The desired Hamilton canonical equations of motion are then obtained as

$$
\begin{aligned}
& \frac{\partial H(\boldsymbol{\xi}, \mathbf{p}, t)}{\partial p_{i}}=\dot{\xi}_{i} \quad(i=1, \ldots, n) \\
& \frac{\partial H(\boldsymbol{\xi}, \mathbf{p}, t)}{\partial \xi_{i}}=\dot{p}_{i} \quad(i=1, \ldots, n)
\end{aligned}
$$

The equation (7) is the set of first-order equations that are particularly symmetrical in form. Hamilton's principle has not provided any new physical theories, but it is sometimes a convenient approach to solve multibody dynamics.

\subsection{Gibbs-Apell equations}

Gibbs-Appell introduced a function known as Gibbs function for the formulation of the equations of motion. This function deals with acceleration, velocity and position. For a system consisting of $N$ particles with mass $m_{i}(i=1, \ldots, N)$ this function is defined as 


$$
G=\frac{1}{2} \sum_{i=1}^{N} m_{i} \dot{\mathbf{r}}_{i}^{2}
$$

where $\ddot{\mathbf{r}}_{i}$ is the acceleration of the $i^{\text {th }}$ particle. The equations of motions are then given by

$$
\frac{\partial G}{\partial \ddot{\xi}_{\mathrm{k}}}=f_{k}
$$

where $\xi_{k}$ is the $k^{t h}$ generalized coordinate, $f_{k}$ represents the $k^{\text {th }}$ generalized force.

Gibbs-Appel equation does not introduce any new physics in classical mechanics but may be more convenient in some cases, particularly when nonholonomic constraints are involved.

\subsection{Kanes equations}

Some methods for the formulation of equations of motion are more efficient than others. Kane's equations [9] enable derivation of the explicit form of equations of motion in a relatively simple and automated way. General form of Kane's equations for a system of rigid-bodies formulated in $N$ generalized coordinates $\xi$ is given by

$$
f_{k}+f_{k}^{*}=f_{k}^{c}, \quad k=1, \ldots, N
$$

where $f_{k}, f_{k}^{*}, f_{k}^{c}$ denote the generalized active, inertia, and constraint forces respectively.

Using the kinematics of multibody system in matrix form and the partitioning of generalized coordinates it is possible to obtain the final representation of the equations in an automated way ready for computer implementation

$$
\mathbf{A} \ddot{\xi}+\mathbf{B} \dot{\xi}+\mathbf{C} \dot{\xi}=\mathbf{F}(t)-\mathbf{J}^{\mathrm{T}} \lambda
$$

where matrices $\mathbf{A}, \mathbf{B}$, and $\mathbf{C}$ contain the contribution from both translational and rotational degrees of freedom. Matrices $\mathbf{B}$ and $\mathbf{C}$ also account for all the quadratic velocity terms, Coriolis forces and nonlinear terms associated with the velocity contribution to the equations. $\mathbf{F}$ is the vector of generalized external forces. $\mathbf{J}$ is the constraint Jacobi matrix, and $\lambda$ is the vector of the Lagrange multipliers.

\section{Modelling of forces in multibody system}

\subsection{Generalized active forces}

Active forces come from pantograph drive system, aerodynamic effects, and contact force as a result of the interaction of collector strips with the overhead contact line. Figure 3 shows a separated link (it can be a part of pantograph) subjected to external forces $\mathbf{F}_{1}$ and $\mathbf{F}_{2}$.

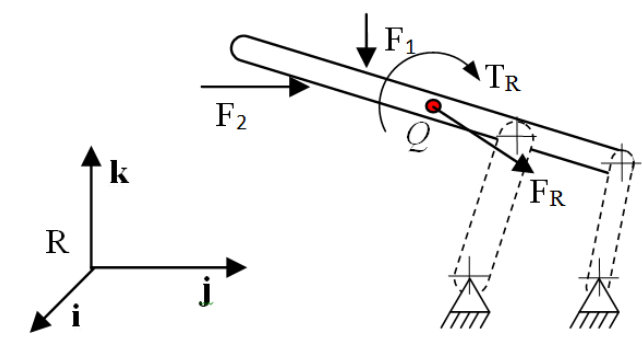

Fig.3. Forces and torques acting on rigid-body.

The equivalent force system can be replaced by the resultant vector force $\mathbf{F}_{\mathrm{R}}$ acting at a point $Q$ (center of gravity) and a couple vector torque $\mathbf{T}_{\mathrm{R}}$. The generalized active force $f_{k}$ associated with a rigid body is given by

$$
f_{k}=\mathbf{F}_{R} \cdot \frac{\partial \mathbf{v}_{k}}{\partial \dot{\xi}_{k}}+\mathbf{T}_{R} \cdot \frac{\partial \boldsymbol{\omega}_{k}}{\partial \dot{\xi}_{k}}
$$

where $\mathbf{v}_{k}$ and $\boldsymbol{\omega}_{k}$ are the vectors of mass center velocity and angular velocity respectively of $k^{\text {th }}$ body in the inertial reference frame. The generalized active force involves both the forces and torques applied on bodies of the system.

\subsection{Modelling of springs and dampers}

Rigid bodies can be connected by springs and dampers in translational motion (Fig.4a) or in rotational motion (Fig.4b).

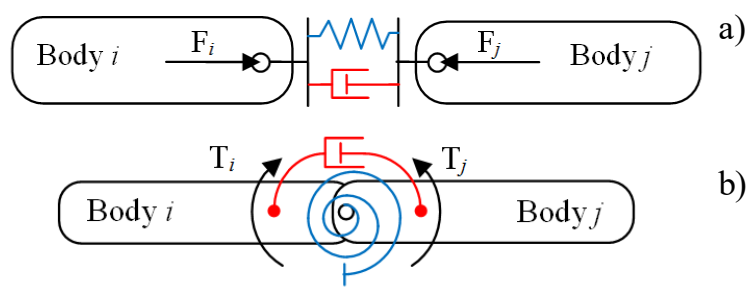

Fig. 4. Spring and damper connecting the rigid bodies in translational motion a) and rotational motion b).

Operation of the linear spring and damper between $i^{\text {th }}$ and $j^{\text {th }}$ bodies can be replaced by equivalent forces $\mathbf{F}_{i}$ and $\mathbf{F}_{j}$ equal in magnitude and opposite in direction.

$$
\mathbf{F}_{i}=F(x, \dot{x}) \mathbf{n}=-\mathbf{F}_{j}
$$

where $\mathbf{n}$ is the unit vector along the axis of the spring, $x$ is the axial deformation of the spring, and $F(x, \dot{x})$ is the function of physical properties of the linear spring and damper. Operation of the rotational spring and damper between $i^{\text {th }}$ and $j^{\text {th }}$ bodies can be replaced by equivalent torques $\mathbf{T}_{i}$ and $\mathbf{T}_{j}$ equal in magnitude and opposite in direction.

$$
\mathbf{T}_{i}=T(\varphi, \dot{\varphi}) \mathbf{n}=-\mathbf{T}_{j}
$$

where $\mathbf{n}$ is the unit vector of torque, $\varphi$ is the relative angle of rotation of the spring, and $T(\varphi, \dot{\varphi})$ is the function of physical properties of the rotational spring and damper. 


\subsection{Gravitational and contact forces}

The forces of gravity acting on a system of $N$ interconnected rigid bodies are usually given by

$$
\mathbf{F}_{i}^{g}=m_{i} g \mathbf{n} \quad(i=1, \ldots, N)
$$

where $n$ is the unit vector of the gravity direction, $m_{i}$ is the mass of $i^{\text {th }}$ body.

The contact forces rise from contact of two rigid surfaces. The contribution of the contact forces to the generalized active forces is zero.

\subsection{Inertia forces}

The inertia forces $\mathbf{F}^{*}$ and inertia torques $\mathbf{T}^{*}$ are generated when the acceleration of the body is not equal to zero. These forces and torques resulting directly from D'Lambert principle

$$
\mathbf{F}^{*}=\sum_{i=1}^{n} m_{i} \mathbf{a}_{i}, \quad \mathbf{T}^{*}=\sum_{i=1}^{n} m_{i} \mathbf{r}_{i} \times \mathbf{a}_{i}, \quad(i=1, \ldots, n)
$$

where the rigid body is assumed to be formed of $m_{i}$ particles, $\mathbf{r}_{i}$ defines the position vector from the point mass to the mass center of the body, and $\mathbf{a}_{i}$ is the acceleration of point mass.

\section{Pantograph dynamic analysis in CAD/CAE application}

Novel analysis methods of multibody dynamics implemented in CAD/CAE applications give the ability to apply forces to pantograph assembly, which allow thorough evaluation of a pantograph performance. Almost all methods for the formulation of the equations of motion can be implemented in computer CAD/CAE applications. Some methods however are more efficient for numerical implementation. Several computer programs (Solidworks, Solid Edge, Autodesk Inventor, etc.) implement multibody dynamics. In each CAD/CAE environment the process of creating the dynamic simulation study of the pantograph involves following steps [10]:

- creating a pantograph assembly,

- creating joints between links (components/parts),

- creating environmental conditions,

- solving equations and analysis of results.

In this work the dynamic simulation workflow implemented in "Dynamic Simulation Module" (DSM) of Autodesk Inventor will be described.

\subsection{Creating an assembly}

An assembly of pantograph is needed in order to create kinematic chains (open and closed) and the physical properties of particular bodies. An example 3D computer model of the 160EC type pantograph is shown in Fig. 1. The Dynamic Simulation Module uses the bodies to convey mass, inertia and centers of gravity information to the multibody dynamics solver.

\subsection{Modelling joints}

There are standard and nonstandard joints in DSM. The selected standard joints are presented in Table 1.

Table 1. Selected standard joints in DSM of Inventor software. DOF means degrees of freedom.

\begin{tabular}{|c|l|l|c|}
\hline Icon & \multicolumn{1}{|c|}{ Name } & \multicolumn{1}{|c|}{ Description } & DOF \\
\hline & Revolution & $\begin{array}{l}\text { No translation } \\
\text { Rotation about the axis }\end{array}$ & 1 \\
\hline & Prismatic & $\begin{array}{l}\text { Translation along the line. } \\
\text { No rotation }\end{array}$ & 1 \\
\hline & Sylindrical & $\begin{array}{l}\text { Translation along the axis } \\
\text { Rotation about the axis }\end{array}$ & 2 \\
\hline & Slanar & $\begin{array}{l}\text { No translation } \\
\text { Rotation about 3 axis } \\
\text { Rotation about the axis }\end{array}$ & $\begin{array}{l}\text { Translation along 3 axis } \\
\text { Rotation about 3 axis }\end{array}$ \\
\hline
\end{tabular}

The nonstandard selected joints grouped into the rolling, sliding, and additional joints are presented in Table 2, Table 3 , and Table 4 respectively.

Table 2. Selected rolling joints in DSM of Inventor software.

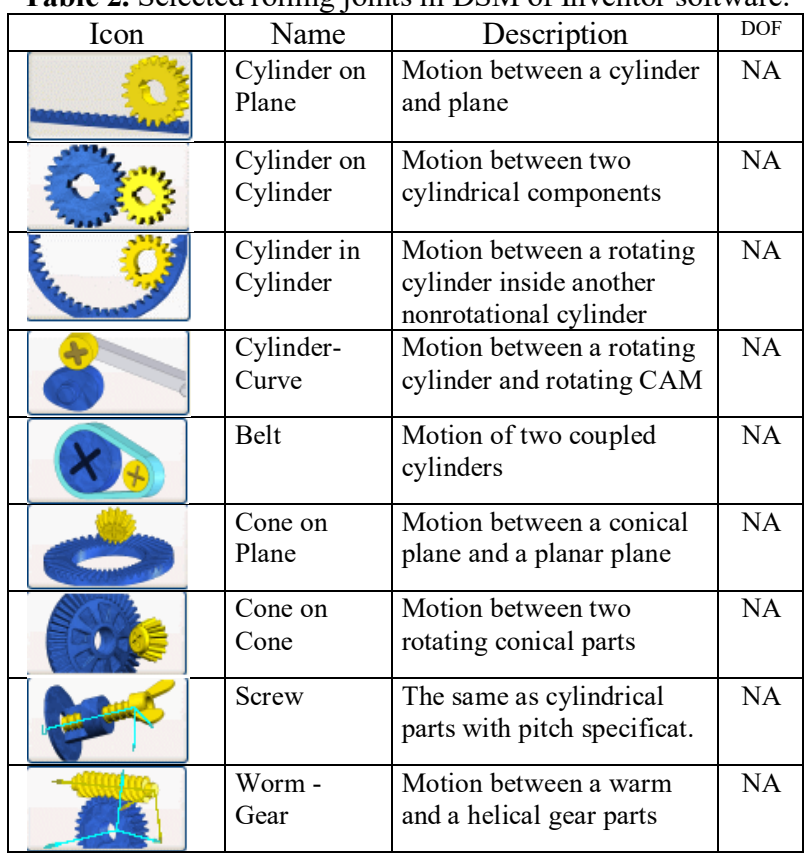

\begin{tabular}{|c|c|c|c|}
\hline Icon & Name & Description & DOF \\
\hline & $\begin{array}{l}\text { Cylinder on } \\
\text { Plane }\end{array}$ & $\begin{array}{l}\text { Sliding between a } \\
\text { nonrotat. cylinder and } \\
\text { plane }\end{array}$ & NA \\
\hline & $\begin{array}{l}\text { Cylinder on } \\
\text { Cylinder }\end{array}$ & $\begin{array}{l}\text { Sliding between two } \\
\text { nonrotating cylinders }\end{array}$ & NA \\
\hline & $\begin{array}{l}\text { Cylinder in } \\
\text { Cylinder }\end{array}$ & $\begin{array}{l}\text { Sliding between a } \\
\text { nonrotat. cylinder inside } \\
\text { another norotatational } \\
\text { cylinder }\end{array}$ & NA \\
\hline & $\begin{array}{l}\text { Cylinder- } \\
\text { Curve }\end{array}$ & $\begin{array}{l}\text { Motion between nonrotat. } \\
\text { cylinder and a rotating } \\
\text { cam }\end{array}$ & NA \\
\hline
\end{tabular}

Table 3. Selected sliding joints in DSM of Inventor software. 
Table 4. Additional joints in DSM of Inventor software.

\begin{tabular}{|l|l|l|l|}
\hline Icon & Name & Description & DOF \\
\hline & $\begin{array}{l}\text { Spring/ } \\
\text { Damper/Jack }\end{array}$ & $\begin{array}{l}\text { This enables to create } \\
\text { springs, dampers and } \\
\text { jacks }\end{array}$ & NA \\
\hline 3D Contact & $\begin{array}{l}\text { This enables to create } \\
\text { contact between two } \\
\text { parts }\end{array}$ & NA \\
\hline
\end{tabular}

When a joint is created there is the possibility to edit:

- initial conditions of starting position and velocity,

- imposed motion to drive the model kinematically by specifying a constant or varying position, velocity and acceleration using the 'Imposed Motion' option as shown in the Fig. 5 ,

- force or torque (in case of a rotation), visco-elastic and dry friction parameters as shown in Fig.6.
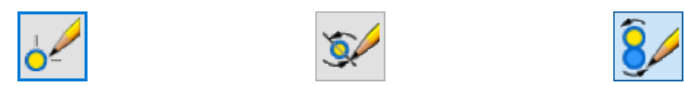

$\square$ Enable imposed motion

$$
\begin{aligned}
& \text { Driving } \\
& \text { Position } \\
& \text { Ovelocity } \\
& \text { Acceleration }
\end{aligned}
$$

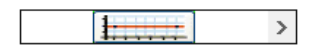

Fig. 5. Defining the imposed motion in a joint.
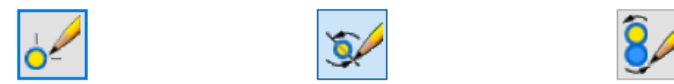

$\square$ Enable joint torque

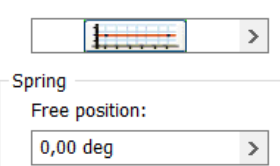

Damping:

Dry friction

Fig. 6. Editing the imposed force or torque and visco-elastic parameters in a joint.

When assembly constraints are converted to joints it is possible to obtain over constrain the assembly at some joints. The mechanism to determine redundancies in joints is implemented in DSM. All joints are visible in the browser which enables the user to recognize them in the model (Fig.7) and edit their parameters.

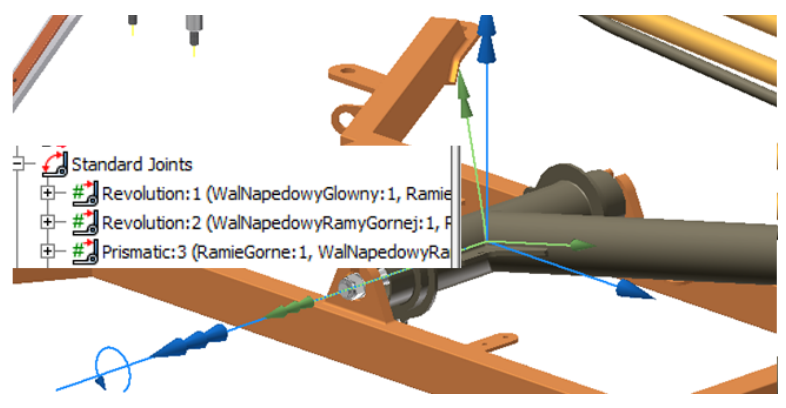

Fig. 7. Set of joints visible in the browser and corresponding local coordinate systems representing joint in the model.

\subsection{Creating environmental conditions}

Once the joints are created and parameters are defined the next step is to apply external loads. The forces and torques need a location on a body to indicate where they are applied, a direction along which or about which the force and torque is applied and a constant or varying value for the magnitude of the force or torque. Figure 8 shows the definition of torque acting on lower arm of the pantograph as function of angular displacement of the lower arm (1200 $\mathrm{Nm}$ at $\alpha=0 \mathrm{rad}-250 \mathrm{Nm}$ at $\alpha=1,6 \mathrm{rad}$ ).

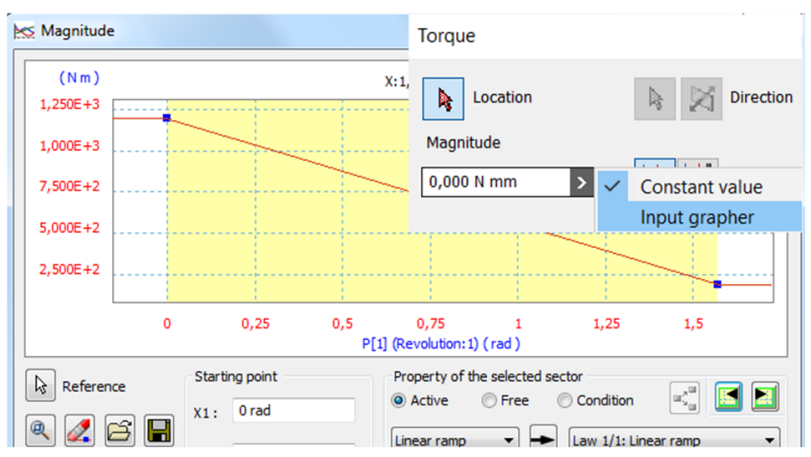

Fig. 8. Characteristic of torque acting on the lower arm as function of angular displacement of this arm.

It is also option to apply the force or torque as a combination of three orthogonal components. The loads can be also defined as functions of position, velocity or acceleration of the body in translational or rotational motion using the 'Input Grapher' editor. The external loads are visible on the pantograph model as vectors indicating the direction and location on the body where they are applied (Fig.9).

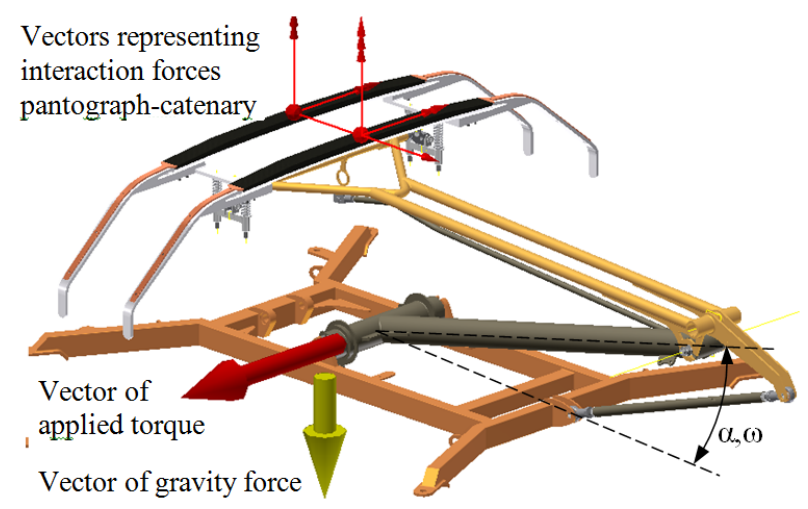

Fig. 9. Visibility of forces and torques as external loads applied to the bodies of the pantograph.

\subsection{Solving and analysis}

The final step is to run the simulation and do the analysis of the results. To run a simulation the user needs to set the simulation time, the number of time steps to record results during the simulation and activate the solver. The simulation results can be analysed in the 'Output Grapher'. The 'Output Grapher' is an interface for reviewing, plotting and post processing the results of a simulation. Waveforms of the angular displacement $\alpha$ and angular velocity $\omega$ of the lower arm are shown in Fig. 10. 


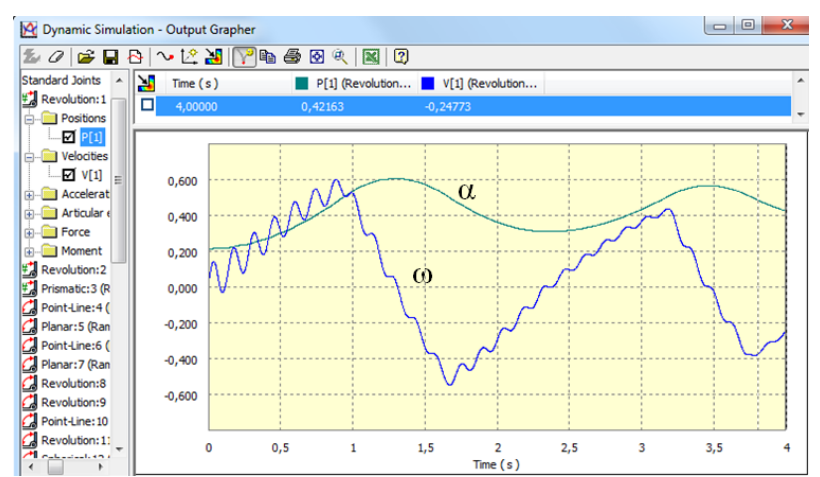

Fig. 10. Results of dynamic simulation presented in the output grapher.

The output grapher gives the possibility to analyse particular joints in terms of: positions, velocities and accelerations; reaction forces and torquies; contact forces; driving forces and torques. The output grapher also enables import and comparison of the results of multiple simulations, export of the results to an excel file, graphs print out.

\section{FEM analysis and dynamic simulation}

The output grapher has functionality to export the simulation results to FEM analysis in Autodesk Inventor Professional for Simulation [11]. This function passes the resultant forces and torques acting on a part and the boundary conditions (based on the joints) to the part FEM analysis module at a given time step during the simulation. Results of FEM analysis on the coupling rod are presented in Fig. 11.

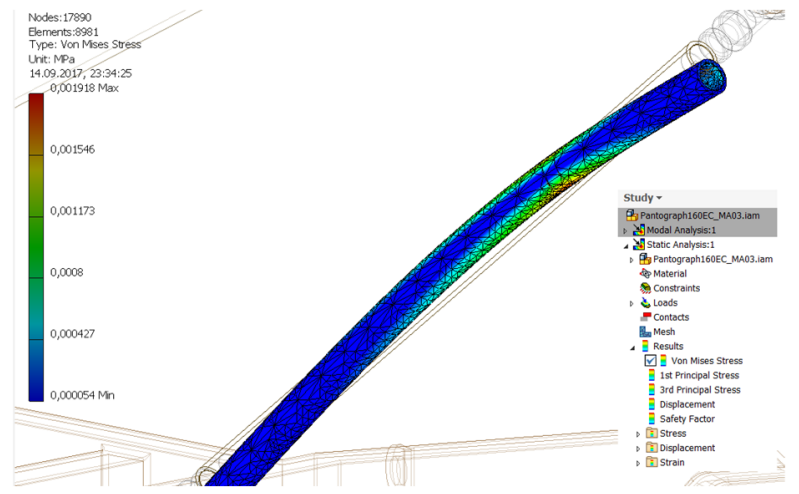

Fig. 11. Von Misses stress results on the coupling rod at some time instant of the dynamic simulation of the pantograph.

\section{Conclusions}

Novel analysis methods of dynamic properties for railway pantographs are implemented in $\mathrm{CAD} / \mathrm{CAE}$ computer applications. Computer applications need numerical implementation of the governing equations of motion. There are several methods for formulation of the equations of motion. Some methods are more efficient for numerical implementation than others. Kane's equations enable to derive the explicit form of equations of motion in a relatively simple and automated way.
Novel analysis methods of multibody dynamics implemented in CAD/CAE applications give the ability to apply "real world" external loads to pantograph assembly, which allow thorough evaluation of pantograph performance. Multibody dynamics in CAD/CAE software enable the analysis of: positions, velocities and accelerations; reaction forces and torquies; contact forces; driving forces and torques in joints. Dynamic simulation is also coupled with FEM calculation.

\section{References}

1. F. Kießling., R. Puschman, A. Schmeider, Contact lines for electric railways. (Publicis Corporate Publishing, Munich Erlanger, 2001).

2. M. Oumri, A. Rachid, A mathematical model for pantograph-catenary interaction, Mathematical and Computer Modelling of Dynamical Systems, 22(5), 463-474 (2016).

3. J. H. Lee, T. W. Park, H. K. Oh, and Y. G. Kim, Analysis of dynamic interaction between catenary and pantograph with experimental verification and performance evaluation in new high-speed line, Vehicle System Dynamics, 53(8), 1117-1134 (2015).

4. S. Bruni, J. Ambrosio, A. Carnicero, Y. H. Cho, L. Finnear, M. Ikeda, S.Y. Kwon, J. P. Massat, S. Stichel, M. Tur, W. Zhang, The results of the pantograph-catenary interaction benchmark, Vehicle System Dynamics, 53(3), 412-435 (2015).

5. F. Amirouche, Fundamentals of multibody dynamics, (Birkhäuser, Boston-Basel-Berlin, 2006).

6. W. Sunada, S. Dubowsky, The Application of FiniteElement Methods to the Dynamic Analysis of Flexible Spatial and Co-planar Linkage Systems, J. Mech. Des., 103(3), 643-651 (1981).

7. M. Kerdjoudj, F. Amirouche, Implementation of the Boundary-Element Method in the Dynamics of Flexible Bodies, International Journal for Numerical Methods in Engineering, 39, 321-354 (1996).

8. A. Wilk, K. Karwowski, S. Judek, M. Mizan, Numerical implementation of current collector model in rotary motion into simulation in translator motion, 12th International Conference Modern Electrified Transport 4-7 October 2015, Trogir, Croatia.

9. T. R. Kane, C. F. Wang, On the Derivation of Equations of Motion, J. Soc. Ind. Appl. Math., 13, (1965).

10. D. Banach, Autodesk Inventor Dynamic Simulation, (Autodesk University, Autodesk Inc 2012).

11. R. Akella, M. Debono, An In-Depth Look at Dynamic Simulation and Stress, Analysis Tools, (Autodesk University, Autodesk Inc 2007). 